Gut, 1968, 9, 414-419

\title{
Site and localization of duodenal ulcers: a study at operation
}

\author{
R. M. KIRK \\ From Hampstead General Hospital, London
}

At operations on 150 patients the duodenal bulb was examined for local features which predispose it to peptic ulceration. A surgeon now has the opportunity to study the duodenum in its natural state and normal situation in both healthy and ulcerated conditions. When gastroenterostomy or partial gastrectomy were routinely used for the cure of duodenal ulcers, the bulb was either left unopened or destroyed. The use of Heineke-Mikulicz pyloroplasty or mucosal antrectomy (Kirk, 1965, 1966) in the treatment of peptic ulcers allows careful internal inspection of the duodenal bulb.

Postmortem studies (Hurst and Stewart, 1929; Portis and Jaffe, 1938) have shown that duodenal ulcers are usually single, near the pylorus, either on the anterior or posterior wall of the bulb. The ulcers are discrete; Doniach and Shiner (1957) did not detect any significant difference between duodenal mucosal biopsy specimens removed from normal and duodenal ulcer subjects. If an excess of hydrochloric acid were the only cause of duodenal ulcers, the whole bulbar chamber, which receives and temporarily retains gastric chyme, should be excoriated, or ulcers should be situated fortuitously. Intensive research has been concentrated on the effects of gastric hypersecretion in the production of duodenal ulcers. In contrast, study of the factors which determine the site and discreteness of the ulcers has been neglected. The theories which have been put forward to explain the site and localization of duodenal ulcers is reviewed below.

\section{THEORIES}

Virchow (1853) argued that if duodenal ulcers are caused solely by acid corrosion they would be generalized and not circumscribed. He invoked a second factor of local ischaemia which determines the site of ulceration.

Wilkie (1911) demonstrated the supraduodenal artery, which he considered to be an end artery; it supplies the area commonly affected by duodenal ulceration. Wilkie thought that obliteration of the lumen of this artery resulted in local ischaemia and consequent increased susceptibility to peptic ulceration. Reeves (1920) reported a relative deficiency in the arterial supply of the first part of the duodenum. Subsequent injection studies by Barclay and Bentley (1949) established the presence of rich anastomoses in the gastric and duodenal walls, with no evidence of end arteries. It appeared unlikely that occlusion of a single vessel would result in local ischaemia. Moreover, numerous experiments have shown that ligation of individual gastric vessels does not produce chronic ulceration, although embolism following the injection of intravascular fat produces acute peptic ulceration (Baronofsky, Merendino, Bratrud, and Wangensteen, 1945).

In the submucosa of the gastric and duodenal walls, arteriovenous anastomoses have been demonstrated which could shunt blood away from the mucosa, producing ischaemia and consequent susceptibility to ulceration (De Busscher, 1948; Barclay and Bentley, 1949). Herzog (1952) demonstrated functioning anastomoses near ulcers. Mucosal blanching has been observed in the stomach (Wolf and Wolf, 1947) but there is no known mechanism which could limit mucosal ischaemia to the usual sites of peptic ulceration.

From his studies with Mann-Williamson dog preparations, Mann (1925) concluded that trauma, from the jet of food and acid emerging through the pylorus impinging on the wall of the bowel beyond, determines the site of peptic ulceration.

An explanation which has not gained wide acceptance is that of Cook (1946). He suggested that external compression of the lesser curvature of the stomach and the duodenal bulb, between the liver and the posterior abdominal wall, determines the site of ulceration.

Taylor and Warren (1956) noted that patients treated conservatively following the perforation of a peptic ulcer remained trouble-free after recovery; patients treated by suture of the perforation commonly developed subsequent chronic ulceration. Taylor and Warren suggested that the insertion of sutures provides a source of foreign body irritation, with increased susceptibility to further ulceration. 


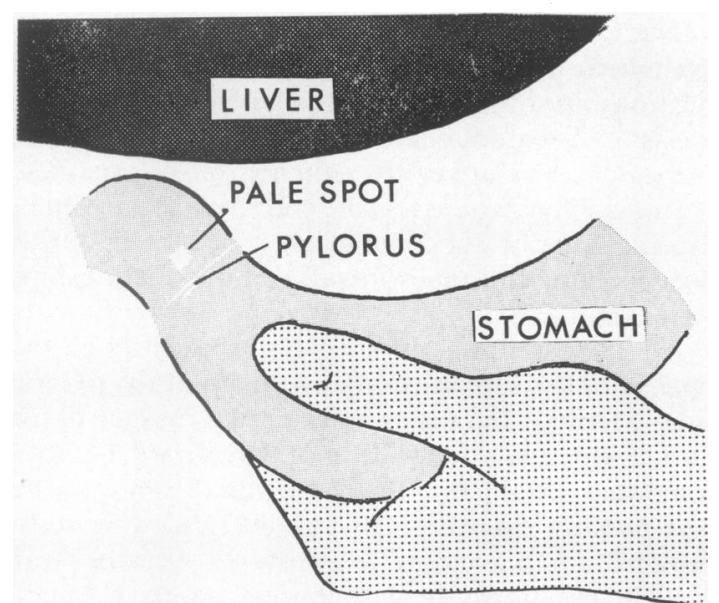

fIG. 1. Mayo's sign. An anaemic spot may appear on the anterior duodenal wall when traction is applied to the distal stomach.

They did not produce evidence that the chronic ulcers developed where the perforated acute ulcers had been sutured.

According to Oi and Sakurai (1959), peptic ulcers occur near the junction between two epithelial cell types. The junction of gastric antral and duodenal mucosae is fairly constant and close to the pylorus, therefore duodenal ulcers usually lie close to the pylorus. If this were the only localizing factor, ulcers should lie fortuitously around the pyloric ring and not be limited to the anterior and posterior walls.

\section{OBSERVATION}

In 1908, Mayo reported that when the duodenal bulb is drawn into the wound by traction on the distal stomach, a white area may appear on the anterior duodenal wall and be mistaken for scarring from a duodenal ulcer (Fig. 1). When the traction is relaxed, the pallor disappears. Mayo recognized the pallor as local ischaemia but did not comment on its possible relation to ulceration. Wilkie (1911) suggested that the pallor resulted from traction on the supraduodenal artery occluding it.

\section{METHODS}

The duodenal bulb was examined at operation in 150 patients.

NORMAL ANATOMY The duodenal bulb was studied in its natural state in 100 patients unaffected by duodenal ulcers or scars.

PATHOLOGICAL ANATOMY While performing vagotomy and mucosal antrectomy for the cure of duodenal ulcers in 50 patients, the inside of the duodenal bulb was examined through the long gastroduodenotomy employed in this technique. The site and size of ulcers and scars were recorded.

TESTS AND EXAMINATION OF LOCALIZING FACTORS The effect of applying N/10 hydrochloric acid to ulcers and normal duodenal mucosa was observed with the naked eye and through a dissecting microscope. The possibility of external compression as a factor in ulcer production was considered. The significance of an acid jet emerging from the pylorus was studied in relation to the production and maintenance of duodenal ulcers. The effect of gastric traction on the patency of vessels supplying the duodenal bulb was observed. Mayo's finding of an anaemic spot on the duodenum produced by exerting gastric traction was re-examined and considered as a possible factor in the localization of duodenal ulcers.

\section{FINDINGS}

NORMAL ANATOMY It was impossible to distinguish the distal extent of the bulb except when the second part of the duodenum contracted, as it did on six occasions. The bulb was then seen to end at the proximal limit of the contracting distal duodenum; there was no evidence of a 'second sphincter' as suggested by Hirschowitz (1961) and Bracey (1961).

No external feature was seen or felt which could explain the usual situation of duodenal ulcers. At gastroduodenotomy on 17 patients unaffected by duodenal ulcers or scars, careful examination of the mucosal aspect also revealed no local features which might determine the site of ulcers.

In view of the reported closeness of ulcers to the pylorus, the depth of the fornices was studied. When the duodenum was drawn towards the surface of the wound by pulling upon the stomach, the fornices were not prominent in 17 patients at gastroduodenotomy, but when traction was relaxed, the pyloric muscle was seen to project markedly into the duodenal bulb. At sphincterotomy operations on three patients, carried out through incisions in the duodenum opposite the ampulla of Vater, a cystoscope was passed proximally to inspect the undisturbed duodenal bulb from within. The depth of the fornices was again noted. Water squeezed from the stomach appeared to remain in the axis of the channel, rather than to strike a particular wall.

The attachments of the duodenal bulb were examined. The bulb was found to be firmly suspended from the underside of the liver by the hepatoduodenal ligament, consisting of innumerable fine strands of inelastic connective tissue running in the right hand portion of the lesser omentum, inserted diffusely into the walls of the duodenal bulb. In 100 patients unaffected by duodenal ulcers or scars, traction on 


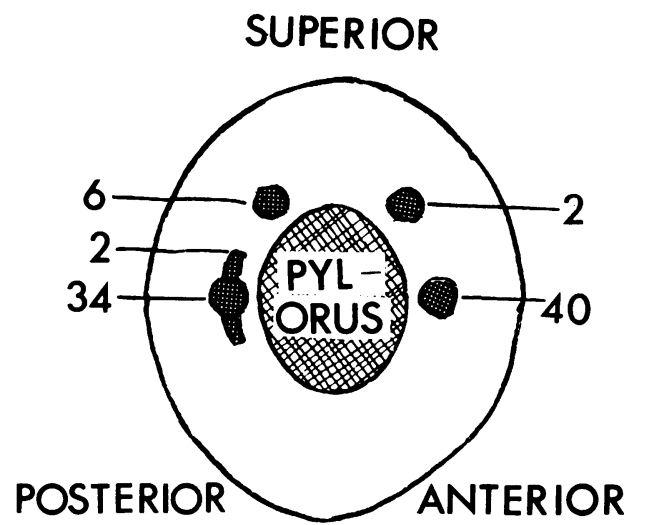

FIG. 2. The situation of ulcers and scars in the duodenal bulb. The diagram shows the duodenal bulb as though inspected from the right side of the patient, after removing the second part of the duodenum.

the bulb did not produce tautness in a particular strand, nor did it pull unequally on any point on the duodenal wall.

PATHOLOGICAL ANATOMY In 50 patients operated upon for the cure of duodenal ulcers, 84 ulcers or scars were seen. There were 36 anterior scars, six anterior ulcers, seven posterior scars, and 35 posterior ulcers.

On the anterior wall, 40 ulcers or scars were midway between the superior and inferior borders, with two lying above the midpoint. On the posterior wall, 36 posterior ulcers or scars were midway between the superior and inferior borders, two of these being linear and parallel to the pylorus; six lay above the midpoint (Fig. 2).

All but one of the ulcers or scars extended proximally to within $1 \mathrm{~cm}$ of the pylorus.

None of the ulcers was as large as $1 \mathrm{~cm}$ in its greatest diameter.

On four occasions, active anterior and posterior ulcers were found in the same patients. Although anterior scars were frequent in the presence of posterior ulcers, in no patient was an anterior ulcer found in the presence of a posterior scar.

TESTS AND EXAMINATION OF LOCALIZING FACTORS In 10 patients locally applied $\mathrm{N} / 10$ hydrochloric acid stimulated a just detectable increase in mucus secretion within two minutes when applied to normal duodenal mucosa after mopping it dry; there was no accompanying colour change.

When acid was applied in six duodenal ulcer craters, no vascular changes were observed. If the acid seeped beyond the ulcer margins on to the surrounding mucosa, an increased secretion of mucus was noted.
The likelihood of external compression was examined. The duodenal bulb is held in a fixed relationship to the under surface of the liver by the inelastic hepatoduodenal ligament. In contrast, the lesser curvature of the stomach is freely mobile.

It was difficult to assess the effect of a jet stream in causing anterior duodenal ulcers, since the bulb was opened along the anterior wall and the relationships were disturbed.

All 35 posterior wall ulcers observed through the opened bulb were overhung by the lip of the pyloric ring. Many of the ulcers were drawn by scar tissue into diverticula, the wide mouths of which faced somewhat distally into the duodenum. Even with the best possible exposure, it was sometimes difficult to demonstrate the ulcers to assistants, because scar fixation had drawn the bases away from the channel.

In all 150 patients the effect of applying gastric traction was observed on the patency of blood vessels supplying the duodenal bulb. In none of them could the palpable arteries, or the visible veins, be occluded by traction. In all the patients, the connective tissue strands or scar tissue became taut before the vessels.

Mayo's finding that gastric traction produces an area of blanching of the anterior duodenal wall at the usual site of anterior ulceration was confirmed in $81 \%$ of 100 patients unaffected by duodenal ulcers or scars. The area was sometimes circular, triangular with the base parallel to the pylorus, linear and parallel to the pylorus, or indefinite in shape (Fig. 3). When traction was applied, the duodenal wall at the anaemic spot could be felt to be under tension greater than that affecting the rest of the duodenal bulb. When gastric traction was relaxed, the pallor disappeared. At gastroduodenotomy on 17 patients unaffected by duodenal ulcers or scars, no pallor of the posterior wall mucosa could be detected when traction was applied. It was not possible to observe the seromuscular aspect of the posterior bulbar wall without disturbing the anatomical relationships. However, when the stomach was drawn downwards and to the left an area of increased tension could be felt on the posterior bulbar wall corresponding with the common site of posterior wall ulcers.

\section{DISCUSSION}

NORMAL ANATOMY No anatomical feature could be detected which might explain the usual situation of duodenal bulbar ulcers. The diffuse attachments of connective tissue which make up the hepatoduodenal ligament cease at the pylorus, and to the left of this the lesser omentum is fragile and elastic. The pyloric ring projects markedly into the duodenal bulb, rather like a spout. 

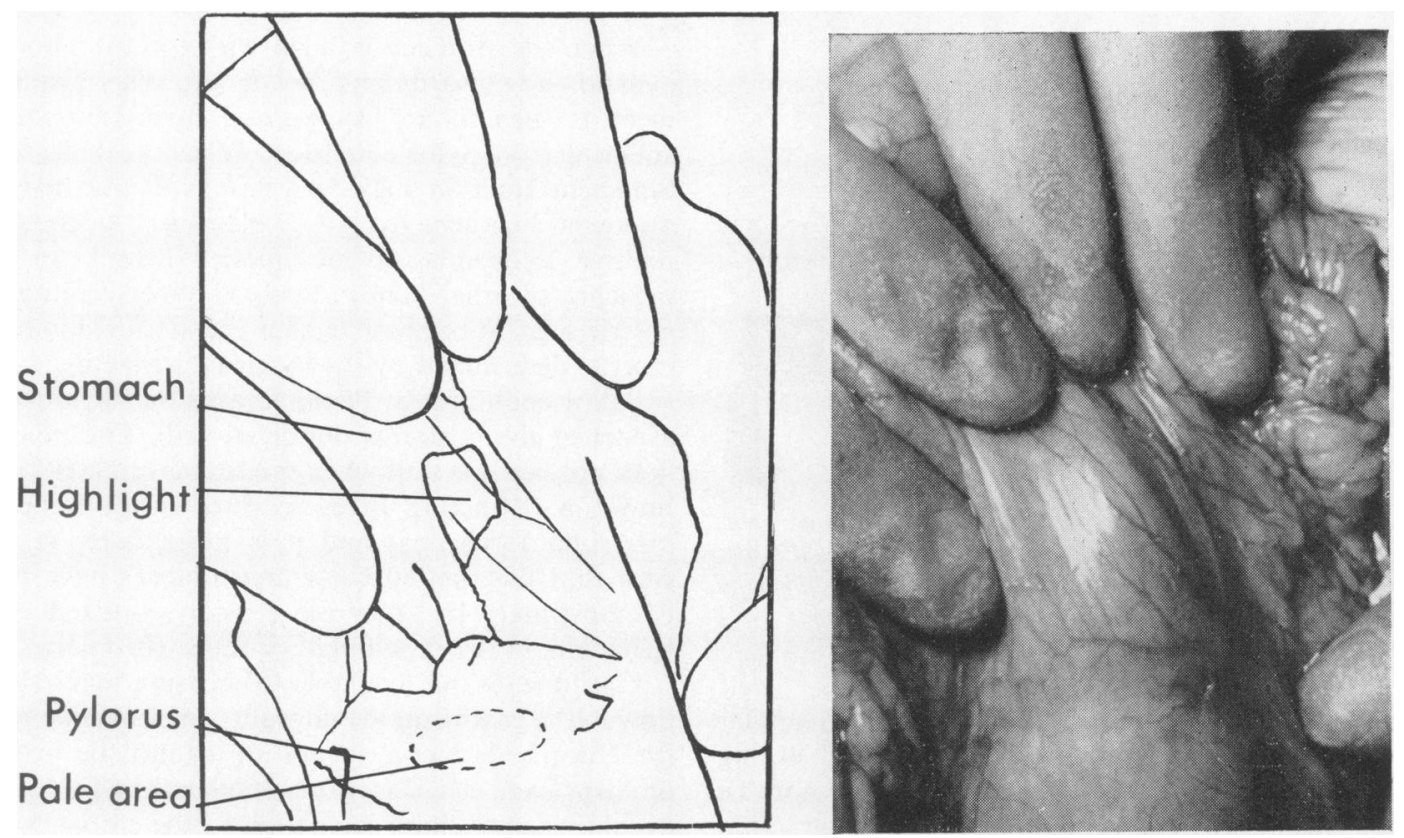

FIG. 3. Unretouched flash photograph of the duodenal bulb, taken while traction was applied to the distal stomach.

PATHOLOGICAL ANATOMY The situation of duodenal ulcers reported in postmortem studies was confirmed at operation. The ulcers lie close to the pylorus, either on the anterior or posterior duodenal walls, usually midway between the superior and inferior borders, occasionally lying superior to the midpoint. Only one ulcer lay at a greater distance than $1 \mathrm{~cm}$ from the pylorus; none of the ulcers was as large as $1 \mathrm{~cm}$ in diameter.

TESTS AND EXAMINATION OF THEORIES The effect of locally applied $\mathrm{N} / 10$ hydrochloric acid was indefinite. Wolf and Wolff (1947)obtained vascular and secretory changes when $\mathrm{N}$ hydrochloric acid was applied to the stomach wall; since this does not occur in physiological or the more usual pathological circumstances, the significance cannot be assessed.

The effect of external compression on the duodenal bulb cannot be dismissed because the hepatoduodenal ligament holds the bulb in a fixed relationship to the liver. Compression of the lesser curve of the stomach is unlikely, because the lax and fragile lesser omentum allows great freedom of movement to the lesser curve. The discreteness of duodenal ulcers makes it difficult to believe that external compression could be sufficiently localized to be responsible.

The effect of an acid jet stream in initiating duodenal ulcers cannot be judged from the findings at operations for the cure of chronic ulcers; the original anatomical relationships may have been destroyed by scarring resulting from ulceration.

The spout-like projection of the pyloric ring in patients unaffected by ulcers makes it appear unlikely that an emerging jet would strike the proximal wall of the duodenal bulb at the usual sites of ulcers. A jet from such a spout would be expected to impinge on the bulbar wall at a distance from the pyloric ring.

The coexistence of active, discrete, anterior and posterior duodenal ulcers on four occasions, without any anatomical feature which could cause splitting of the jet stream, argues powerfully against the maintenance of ulcers by the trauma of a jet stream. Hurst and Stewart (1929) reported finding 27 subjects with active anterior and posterior duodenal ulcers at postmortem examination.

All the 35 posterior wall chronic ulcers lay close to the pylorus out of the direct line of an emerging jet stream. It is difficult to visualize acid and food expelled from the stomach always striking the same small area, producing and maintaining such discrete, small ulcers as were seen.

Wilkie's suggestion that duodenal traction produces occlusion of supplying vessels was not confirmed. Always the connective tissues became taut, leaving the vessels patent.

Mayo's finding that an area of blanching may appear on the unscarred anterior duodenal wall when gastric traction is applied, was confirmed in $81 \%$ of 100 patients. The blanching was associated with 


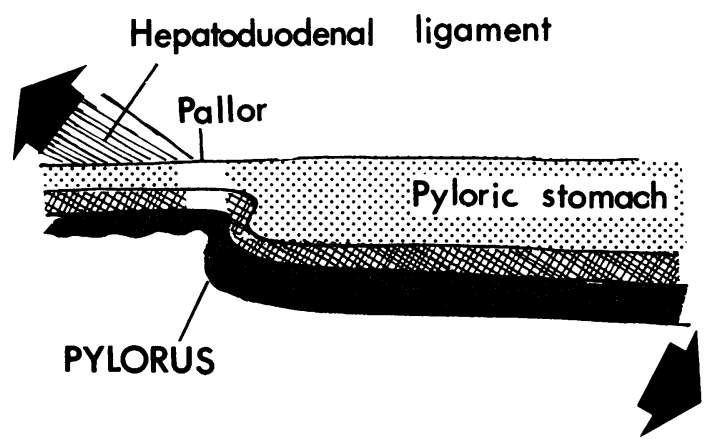

FIG. 4. Suggested mechanism of Mayo's anaemic spot. Traction on the thick-walled distal stomach causes distraction of the proximal duodenal wall which is supported distally by the fibres of the hepatoduodenal ligament.

localized tension felt in the duodenal wall. A similar tension could be felt in the posterior wall during gastric traction, although pallor could not be detected. The tension seemed to be due to distraction of a small area of duodenal wall between the pull on the one hand of the hepatoduodenal ligament, and the pull exerted on the thick-walled gastric antrum (Fig. 4). The intervening thin-walled segment of duodenum became blanched because the tension in the tissues squeezed the blood vessels empty. The localized pallor was produced in a manner similar to that seen in the skin over the dorsum of the metacarpophalangeal joints of the hand when making a fist.

\section{HYPOTHESIS}

Confirmation of Mayo's finding, that traction on the distal stomach frequently produces an anaemic spot on the anterior duodenal wall where ulcers are usually found, suggests a link between the localized anaemia and the site of duodenal ulcers.

The mechanistic concept of the stomach hanging in the peritoneal cavity, slung like a hammock between the gullet and the hepatoduodenal ligament, is no longer accepted. However, there are two circumstances in which the stomach may descend within the abdomen and so apply traction on the hepatoduodenal ligament.

The specific gravity of the abdominal viscera is 1.03 to 1.06 (Schäfer and Symington, 1898); if the stomach is filled with material of higher specific gravity than this, it sinks. Conventional contrast media used in barium meal radiological examinations $(1.4$ to $2.0 \mathrm{~g} / \mathrm{cmm}$, Sandmark, 1963) act in this way.
When the stomach is filled with food or fluid, it expands downwards and to the left. This displacement is seen in an exaggerated form in patients suffering from pyloric stenosis and gastric distension. Sufficient traction may be exerted on the hepatoduodenal ligament by this displacement to produce relative ischaemia of the duodenal wall. In the presence of other factors, such as hypersecretion of gastric acid, it is possible that the site of duodenal ulcer is determined by the localized ischaemia.

Pallor could not be demonstrated on the mucosal aspect of the posterior duodenal wall. The mucosa does not become taut when traction is applied to the bowel: a distracting force is resisted by the collagenous submucous coat and it is particularly in this coat that the rich vascular anastomoses have been demonstrated. If a mucosal erosion occurred, then ischaemia in the base might produce chronicity.

Until means are available to measure local blood flow in the gastro-duodenal walls, an ischaemic basis for chronic duodenal ulceration cannot be proved or disproved. The localized duodenal pallor which occurs when traction is applied to the stomach can be appreciated with the naked eye; its coincidence with the usual site of duodenal ulcers suggests that the ischaemia and ulcers are related as causal agent and effect.

If this hypothesis is a correct interpretation of the findings, then there seems to be some merit in advising duodenal ulcer patients to eat frequent small meals and so prevent gastric distension and displacement.

\section{SUMMARY}

The duodenal bulb was studied at operation in 50 patients affected by duodenal ulceration and in $\mathbf{1 0 0}$ unaffected patients. Duodenal ulcers were confirmed to lie close to the pylorus, either on the anterior or posterior duodenal walls, usually midway between the superior and inferior borders. No anatomical feature was detected which could explain the site and localization of duodenal ulcers. N/10 hydrochloric acid applied locally to normal mucosa and into ulcer bases gave an indefinite response. External compression of the duodenal bulb is an unlikely localizing factor for ulceration.

The effect of trauma from an acid jet stream emerging through the pylorus and striking the duodenal wall could not be assessed in the initial causation of duodenal ulcers. A jet emerging through the spout-like pylorus would be unlikely to impinge on the proximal duodenal wall at the usual site of ulceration. Chronic ulcers appeared to lie well out of the line of an acid jet stream, being drawn away 
by the scarring resulting from ulceration. The finding of discrete active anterior and posterior duodenal ulcers in four patients who had apparently normal pyloric channels argues against the jet theory of causation.

Duodenal traction does not occlude the blood vessels supplying the duodenal bulb, as Wilkie suggested.

The observation by Mayo that traction on the stomach may cause an area of pallor on the anterior duodenal wall at the usual site of anterior duodenal ulcers, was confirmed in $81 \%$ of 100 patients unaffected by duodenal ulcers or scars. Gastric traction produced a local rise in duodenal tissue tension, with resulting emptying of the small vessels.

Filling the stomach with food may produce sufficient gastric displacement and consequent duodenal traction to give an ischaemic area more susceptible to acid attack.

Tension on the bowel wall is resisted by the submucous coat and it is in this coat that the rich vascular anastomoses have been demonstrated. Traction may render an area ischaemic by squeezing the blood vessels empty, however rich the general blood supply. Local tension results from distraction of a small segment of duodenal wall between the diffusely inserted hepatoduodenal ligament on the one hand and the thick-walled pyloric antrum on the other. Tension was felt, but ischaemia could not be observed, at the point on the posterior duodenal wall corresponding with the usual site of posterior ulcers. Although mucosal pallor was not seen when traction was applied, it is possible that an erosion is converted into a chronic ulcer if there is ischaemia in the base.

\section{REFERENCES}

Barclay, A. E., and Bentley, F. H. (1949). The vascularisation of the human stomach. A preliminary note on the shunting effect of trauma. Brit. J. Radiol., 22, 62-67.

Baronofsky, I., Merendino, K. A., Bratrud, T. E., and Wagensteen, O. H. (1945). Fate of intravenously injected fat: its role in the production of ulcer. Proc. Soc. exp. Biol. (N.Y.), 59, 231-234.

Bracey, D. W. (1961). The fiberscope. Lancet, 1, 1223-1224.

de Busscher, G. (1948). Etude morphologique et considérations physiologiques sur la vascularisation de l'estomac. Acta gastro-ent. belg., 11, 333.

Cook, D. (1946). Ulcer: The Primary Cause of Gastric and Duodenal Ulcer. Medical Center Foundation, Chicago.

Doniach, I., and Shiner, M. (1957). Duodenal and jejunal biopsies. II. Histology. Gastroenterology, 33, 71-86.

Herzog, W. (1952). Zur Gefässhistologie das Magens und Zöwlffingerdarmes beim Ulcus ventriculi and duodeni. Bruns' Beitr. klin. Chir., 184, 74-84.

Hirschowitz, B. I. (1961). Endoscopic examination of the stomach and duodenal cap with the fiberscope. Lancet, 1, 1074-1078.

Hurst, A. F., and Stewart, M. J. (1929). Gastric and Duodenal Ulcer. Oxford University Press.

Kirk, R. M. (1965). Mucosal antrectomy with vagotomy in the treatment of duodenal ulcer. Brit. J. Surg., 52, 604-606.

- (1966). Mucosal antrectomy in the treatment of peptic ulcer. Proc. roy. Soc. Med., 59, 571-575.

Mann, F. C. (1925). The chemical and mechanical factors in experimentally produced peptic ulcer. Surg. Clin. N. Amer., 5, 753-775.

Mayo, W. J. (1908). Anemic spot on the duodenum which may be mistaken for ulcer. Surg. Gynec. Obstet., 6, 600-601.

Oi, M., and Sakurai, Y. (1959). The location of duodenal ulcer. Gastroenterology, 36, 60-64.

Portis, S. A., and Jaffé, R. H. (1938). A study of peptic ulcer based on necropsy records. J. Amer. med. Ass., 110, 6-13.

Reeves, T. B. (1920). A study of the arteries supplying the stomach and duodenum and their relation to ulcer. Surg. Gynec. Obstet., 30, 374-385.

Sandmark, S. (1963). Hiatal incompetence. Acta radiol. Diagn. (Stock.), suppl. 219, 28.

Schäfer, E. A., and Symington, J. (1898). In Quain's Elements of anatomy. vol. 3, pt. 4. Edited by E. A. Schäfer and G. D. Thane. Longmans, London.

Taylor, H., and Warren, R. P. (1956). Perforated acute and chronic peptic ulcer. Conservative treatment. Lancet, 1, 397-399.

Virchow, R. (1853). Einfaches chronisches Magengeschwür. Virchows Arch. path. Anat., 5, 362-364.

Wilkie, D. P. D. (1911). The blood supply of the duodenum. Surg. Gynec. Obstet., 13, 399-405.

Wolf, S., and Wolff, H. G. (1947). Human Gastric Function. 2nd ed. Oxford University Press, London. 